\title{
Reduction of radiation dose for coronary computed tomography angiography using prospective electrocardiography-triggered high-pitch acquisition in clinical routine
}

\author{
Narumol Chaosuwannakit ${ }^{1 A, B, C, D, F, F}$, Pattarapong Makarawate ${ }^{2 E}$ \\ 'Department of Radiology, Faculty of Medicine, Khon Kaen University, Khon Kaen, Thailand \\ ${ }^{2}$ Cardiology Unit, Department of Internal Medicine, Faculty of Medicine, Khon Kaen University, Khon Kaen, Thailand
}

\section{Abstract}

Purpose: To evaluate the image quality, radiation exposure, and means of application in a group of patients who underwent coronary computed tomography angiography (CCTA) performed with low-dose prospective electrocardiography (ECG)-triggered acquisition in which a standard sequence was added if the low-dose sequence did not allow reliable exclusion of coronary stenosis with respect to image quality.

Material and methods: The present study was approved by the Ethics Committee of the Faculty of Medicine, and informed consent was obtained from all patients. The authors performed a retrospective review of 256 consecutive patients referred for CCTA using dual-source CT scanner (Definition FLASH, Siemens, Germany). CCTA was performed using prospective ECG-triggered high-pitch acquisition. In patients with higher heart rates (> $65 \mathrm{bpm})$ or in whom irregular heart rates were noted prior to the scan, a subsequent CCTA was performed immediately (double flash protocol). The effective radiation dose was calculated for each patient. All images were evaluated by two independent observers for quality on a four-point scale with 1 being non-diagnostic image quality and 4 being excellent.

Results: Mean effective whole-body dose of CCTA was $1.6 \pm 0.4 \mathrm{mSv}$ (range, 0.4-5.4) for the entire cardiac examination and $0.9 \pm 0.3 \mathrm{mSv}$ (range, 0.4-2.8) for individual prospective ECG-triggered high-pitch CCTAs. In 27 of these patients with higher heart rates or occasional premature ventricular contractions or atrial fibrillation, subsequent CCTAs were performed immediately. The average image quality score was good to excellent with less than $1 \%$ unevaluable coronary segments. The double flash protocol resulted in a fully diagnostic CCTA in all cases.

Conclusions: The prospective ECG-triggered high-pitch CCTA technique is feasible and promising in clinical routine with good to excellent image quality and minimal radiation dose. The double flash protocol might become a more robust tool in patients with higher heart rates or arrhythmia.

Key words: radiation exposure, coronary CTA, image quality, computed tomography.

\section{Introduction}

Coronary computed tomography angiography (CCTA) has increasingly gained importance as a noninvasive, fast, and accurate study for diagnosing coronary artery disease (CAD) [1-7]. The relatively high radiation exposure in CCTA compared with invasive conventional coronary angiography remains a challenge $[8,9]$. Hence, not only careful selection of patients suitable for CCTA examination, but also reduction of the patient radiation exposure in CCTA without compromising diagnostic accuracy should be aimed for. CCTA performed with 64-detector CT without the use of electrocardiography (ECG) pulsing typically results in radiation doses rang-

Correspondence address:

Narumol Chaosuwannakit, MD, Radiology Department, Faculty of Medicine, Khon Kaen University, Khon Kaen, Thailand, 40000, phone: 084-6464648,

e-mail:narumol_chao@yahoo.com

Authors' contribution:

A Study design · B Data collection · C Statistical analysis · D Data interpretation · E Manuscript preparation · F Literature search · G Funds collection 
ing from 15 to $21 \mathrm{mSv}$ [5] and $9 \mathrm{mSv}$ with the use of ECG pulsing [10,11].

It is well known that the radiation dose in cardiac imaging can substantially be reduced by the use of prospective ECG-triggering data acquisitions [12]. The method of prospective ECG-triggering (PT) has been evaluated for image quality of the coronary arteries and diagnostic accuracy as well as effective radiation dose in several studies [13-19]. PT is a technique used with CCTA, which uses forward-looking prediction of R-wave timing, step-andshoot non-spiral acquisition during imaging, and unique cone beam reconstruction [20]. By contrast, CCTA with standard retrospective ECG-triggering (RT) uses backward-looking measurement of R-wave timing, spiral scanning during table motion, and more traditional cone beam reconstruction. In addition, with $\mathrm{PT}$ the X-ray beam is turned on for only a short portion of diastole, and it is turned off during the rest of the R-R cycle, whereas with $\mathrm{RT}$ the X-ray beam is turned on throughout the R-R interval. Initial results showed that PT may be able to provide sufficient image quality with low radiation dose [13-16].

The latest generation of dual-source CT (DSCT) scanners has introduced a new scan mode - a prospective ECG-triggered helical data acquisition with very high pitch values of more than 3.0. This technique enables acquisition of the entire volumetric dataset of the heart within a fraction of a single cardiac cycle. The high pitch allows acquisition with very low radiation exposure $(<1 \mathrm{mSv})$ in patients with low, regular heart rates $[17,18]$. Accurate and consistent visualisation of the coronary arteries at high image quality is critical for coronary CTA. Hence, the purpose of this study was to describe the results of prospective ECG-triggered high-pitch mode for coronary CTA in clinical routine practice in a tertiary care centre with respect to image quality and radiation exposure.

\section{Material and methods}

\section{Patients}

This retrospective study included 256 patients (130 males and 126 females) referred for CCTA between February 2013 and February 2014. The indications for CCTA were an abnormal, equivocal, or non-diagnostic stress test, chest pain, evaluation of cardiomegaly and congestive heart failure, as well as the evaluation of cardiac aetiology of syncope. Patients with an intermediate probability of coronary artery disease (CAD) were also referred for a CCTA as a first test. The above are considered appropriate indications for CCTA, based on the criteria of the American College of Cardiology (ACC) [19-21] and the recent American Heart Association Scientific Statement on Cardiac CT [19-21]. Exclusion criteria for CCTA included the presence of renal failure, pregnancy, and a history of allergic reaction to iodine-containing contrast agents. The present study was approved by the Ethics
Committee of the Faculty of Medicine. Written, informed consent was not required because of the retrospective nature of the investigation.

\section{Cardiac computed tomography angiography protocol}

CCTA examinations were performed on a DSCT scanner (Definition FLASH, Siemens Healthcare, Forchheim, Germany). The system was equipped with two $\mathrm{X}$-ray tubes and two corresponding detectors mounted on a single gantry with an angular offset of $90^{\circ}$ [22]. The CCTA used automatic tube current modulation in $x, y, z$ directions (Care Dose 4D, Siemens Healthcare). The scanner technology enabled a prospectively ECG-triggered high-pitch (3.4) spiral acquisition (FLASH Spiral Cardio, Siemens Healthcare, Forchheim, Germany). The CCTA scan parameters were as follows: two X-ray sources, detector collimation $128 \times 0.6 \mathrm{~mm}$ with double sampling by rapid alteration of the focal spot in the longitudinal direction (Z-flying focal spot) [23], rotation time 0.28 seconds, and tube voltage 100 or $120 \mathrm{kV}$. Image acquisition was performed during inspiratory breath-hold. To familiarise the patient with the protocol, breath-holding was practiced before the examination. A contrast agent bolus of 50-80 $\mathrm{ml}$ was injected with a mean flow rate of $5 \mathrm{ml} / \mathrm{s}$ followed by a $50 \mathrm{ml}$ saline flush. Circulation time from the antecubital fossa to the aortic root was determined in each patient with administration of a timed bolus of $20 \mathrm{ml}$ of iodinated contrast. The entire volume of the heart was covered during one breath-hold in approximately five seconds with simultaneous recording of the ECG trace. Patients were scanned in the supine position twice, first without contrast medium to calculate the calcium score and secondly after contrast medium injection. Studies were acquired in the cranio-caudal direction from the level of the carina to just below the diaphragm.

In 27 out of the 256 patients a second CCTA was acquired immediately during the same contrast injection timed ("double flash protocol"). In these cases, the delay time between the two acquisitions was set to four seconds, the minimal allowable time on this system. Therefore, the total scan time was longer and the total contrast volume was increased by 25 to $35 \mathrm{cc}$ compared to single high-pitch CCTA acquisition ([ 4 seconds $+\sim 1$ second scan] $\times$ flow rate). This protocol was used in patients with higher heart rates ( $>65 \mathrm{bpm}$ ) or in whom irregular heart rate was noted prior to the scan. Neither nitroglycerine nor beta-blockers were given to the patient.

\section{Cardiac computed tomography angiography image reconstruction}

All CCTA datasets were transferred to a dedicated workstation. To evaluate the coronary arteries, the images were reconstructed with a small FOV (120-190 mm), which was restricted to the heart region. The images were reviewed in the curve multiplanar reconstruction, axial, 
Table 1. Four-point rating scale for assessment of image quality. Scores of 3 and 4 correspond to a non-diagnostic image quality that does not allow assessment of the entire coronary artery for the presence of a flow-limiting stenosis

\begin{tabular}{|l|l|}
\hline Score & Characteristics \\
\hline 1 & $\begin{array}{l}\text { Non-diagnostic image quality for multiple segments } \\
\text { within one coronary artery considered unevaluable }\end{array}$ \\
\hline 2 & $\begin{array}{l}\text { Acceptable image quality with artefacts but not } \\
\text { compromising diagnostic vessel assessment }\end{array}$ \\
\hline 3 & $\begin{array}{l}\text { Good image quality with minimal artefacts but fully } \\
\text { evaluable coronary vessels }\end{array}$ \\
\hline 4 & Excellent image quality without artefacts \\
\hline
\end{tabular}

coronal, and sagittal planes, using a mediastinal window (width: 450, level: 35), lung window (width: 1500, level: -700), and bone window (width: 1500, level: 450) for all examinations.

\section{Coronary computed tomography angiography image analysis}

CCTA image analysis was performed by two independent experienced cardiovascular and thoracic radiologists (with a respective nine and seven years of experience in examining cardiovascular and thoracic CT scans) on a dedicated post processing workstation.

Image quality was analysed for every segment according to a four-point scale (Table 1$)[24,25]$ (1 = non-diagnostic image quality; 2 = acceptable image quality with artefacts but not compromising diagnostic vessel assessment; 3 = good image quality with minimal artefacts but fully evaluable coronary vessel structures; $4=$ excellent image quality without artefacts). Typical examples for each grade (1-4) are given in Figures 1 and 2. The two readers rated the image quality independently with a subsequent consent read.

\section{Measurement of the radiation exposure}

The dose-length product (DLP) displayed by the CT unit was recorded for each CCTA. The effective dose of CCTA was estimated by a method proposed by the Fleischner Society [26]. The effective dose is derived from the product of the DLP and a conversion coefficient for the anatomical region examined which is $0.014 \mathrm{mSv} \mathrm{mGy}^{-1} \mathrm{~cm}^{-1}$ for the chest [27]. However, it should be noted that a uniform conversion coefficient for all images is not entirely accurate because it does not account for the different conditions in the examinations.

\section{Statistical analysis}

Statistical analyses were performed using SPSS software version 16 (SPSS, Inc., Chicago, IL, USA). Continuous data were expressed as mean $\pm \mathrm{SD}$, and categorical variables were given as percentages. Comparisons of the patient characteristics, the CT parameters, and the dose estimates were performed using the $t$-test for normally distributed data, and using the $\chi^{2}$ test if not. Intraclass correlation coefficient (ICC) was used for interobserver agreement of subjective image quality. The coefficient represents concordance, where 1 is perfect agreement and 0 is no agreement at all. A significance level of $p<0.05$ was considered a statistically significant result, and all reported $p$-values were two-sided.

\section{Results}

A total of 256 patients (130 men, 126 women) were included in this study over a period of 12 months. Clinical characteristics of the patients are summarised in Table 2. A total of 283 CCTAs in 256 patients were evaluated. In 27 patients two prospectively triggered high-pitch acquisitions were performed. A second CCTA was acquired immediately during the same contrast injection timed
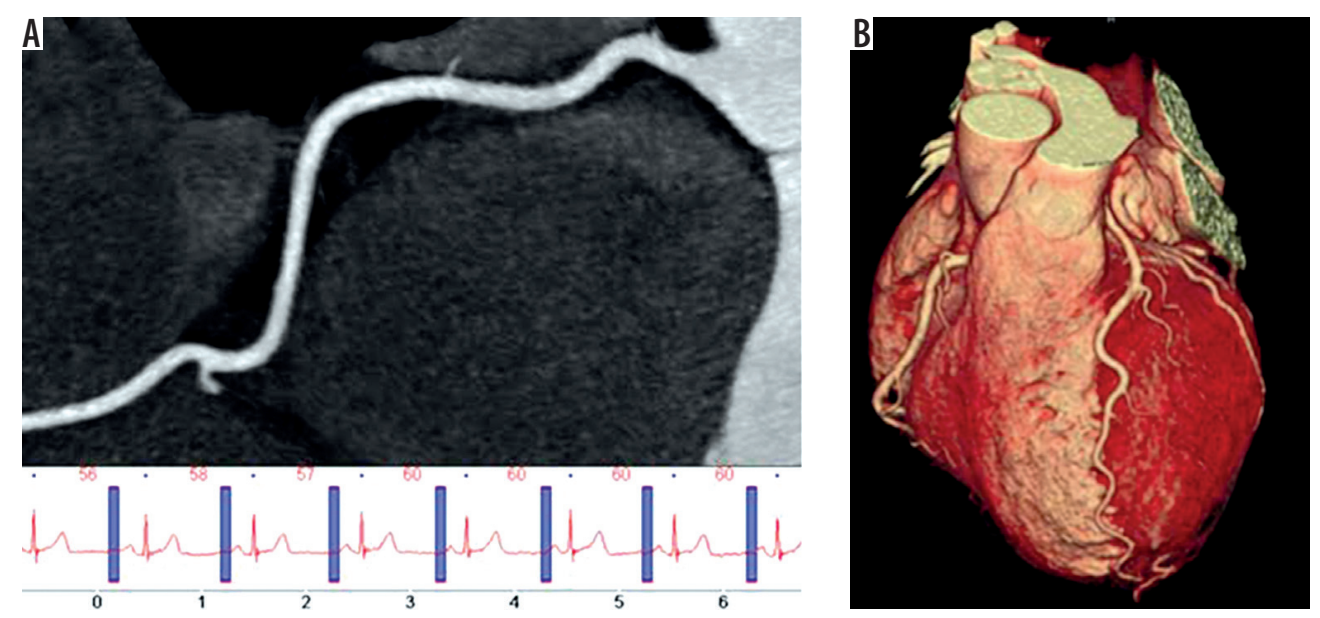

Figure 1. Example of excellent image quality using prospective electrocardiography (ECG)-triggered high-pitch (pitch 3.4) acquisition of a 65-year-old male patient with atypical chest pain. Images show a curved multiplanar reconstruction of the right coronary artery (A) and a 3D volume-rendered reconstruction of coronary arteries (B) demonstrating excellent image quality. Average heart rate is $59 \mathrm{bpm}$, estimated radiation dose is $0.5 \mathrm{mSv}$, and amount of contrast agent is $50 \mathrm{ml}$ 

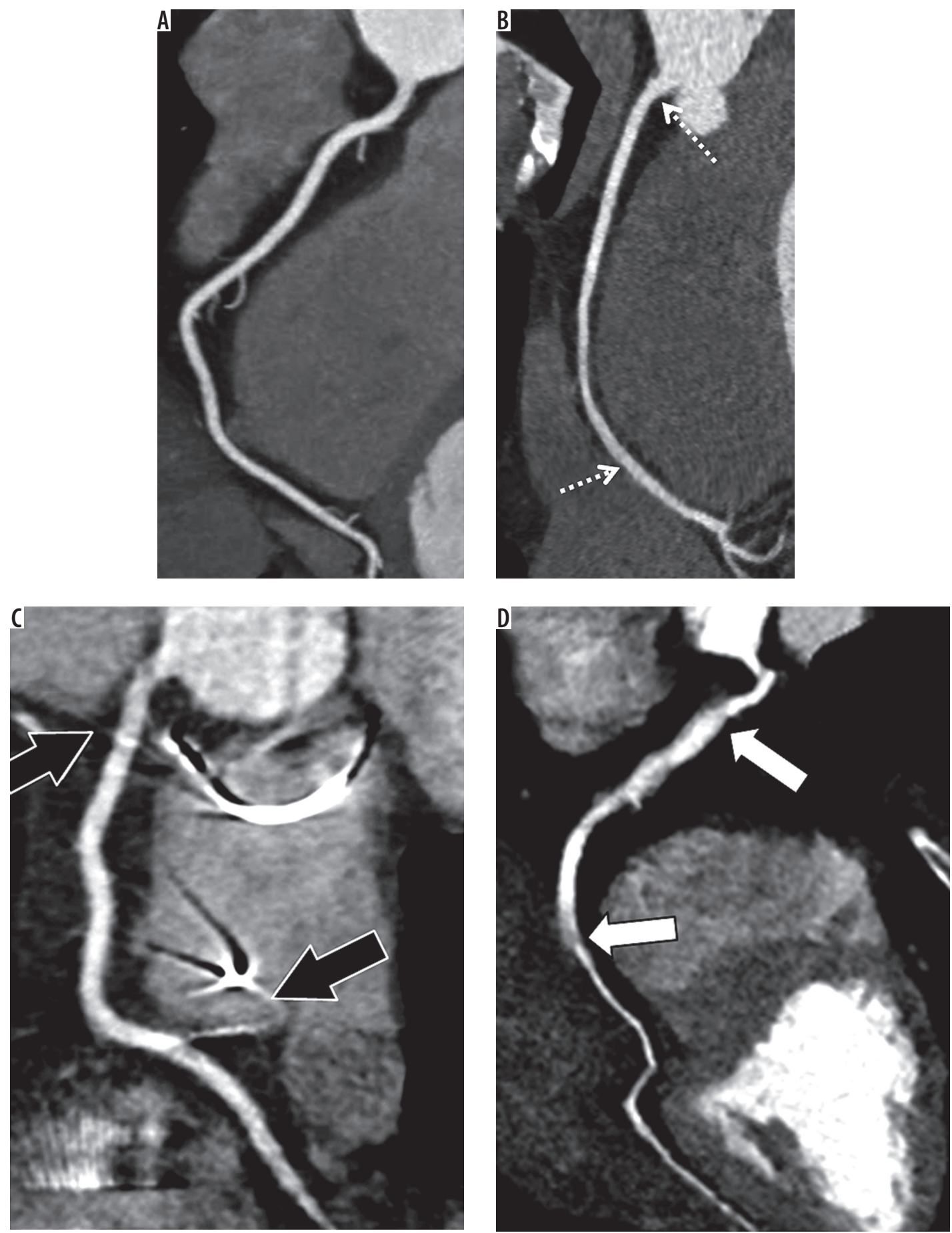

Figure 2. The figure demonstrates typical images for each grade (1-4) regarding the right coronary artery (RCA). A) Excellent image quality (grade 4). B) Good image quality (grade 3) with minimal artefacts but fully evaluable coronary vessel (dash arrows). C) Moderate image quality (grade 2) due to beam hardening artefacts of pacemaker leads (black arrows). D) Severe blurring artefacts (white arrows) cause non-diagnostic image quality (grade 1) of the proximal and mid RCA

(“double flash protocol"). This protocol was used in patients with higher heart rates ( $>65 \mathrm{bpm})$ or in whom irregular heart rates were noted prior to the scan.

Patients in the single prospectively ECG-triggered highpitch group (SP) and those in the double flash protocol (DF) group did not differ significantly in age, sex, or mean body mass index. However, there was a significant difference between the groups for mean heart rate ( $58 \pm 9$ beats/ min for the SP group, $75 \pm 11$ for the DF group; $p=0.02$ )
(Table 2). The distribution of calcium score of all patients are shown in Table 3.

In SP group, the mean DLP was $63.7 \pm 21 \mathrm{mGy} \cdot \mathrm{cm}$, resulting in an effective radiation dose per examination of $0.9 \pm 0.3 \mathrm{mSv}$. In the DF group, mean DLP was 114.3 $\pm 28 \mathrm{mGy} \cdot \mathrm{cm}$, resulting in an effective radiation dose per examination of $1.6 \pm 0.4 \mathrm{mSv}$. The SP group showed a significantly lower DLP and consequently a significantly lower mean effective radiation dose compared with the 
Table 2. Patient characteristics of the single prospective electrocardiography (ECG)-triggered high-pitch coronary computed tomography angiography (SP) and double flash protocol (DF)

\begin{tabular}{|c|c|c|c|}
\hline Characteristic & SP & DF & $p$-value \\
\hline Age (years) ${ }^{*}$ & $54 \pm 10(25-81)$ & $58 \pm 15(45-81)$ & 0.15 \\
\hline Men $^{\#}$ & $130(50.8 \%)$ & $130(50.8 \%)$ & 1.0 \\
\hline Height $(\mathrm{cm})^{*}$ & $161.8 \pm 13$ & $158.9 \pm 15$ & 0.25 \\
\hline Weight $(\mathrm{kg})^{*}$ & $59.8 \pm 12$ & $52.5 \pm 10$ & 0.16 \\
\hline Body mass index $\left(\mathrm{kg} / \mathrm{m}^{2}\right)^{*}$ & $23.1 \pm 6(15-35)$ & $21.2 \pm 8(18-35)$ & 0.41 \\
\hline Heart rate $(\mathrm{bpm})^{*}$ & $58 \pm 9(50-64)$ & $75 \pm 11(65-100)$ & $0.02^{*}$ \\
\hline
\end{tabular}

*Data are mean \pm standard deviation, numbers in parentheses are range.

"Data are numbers of patients, and numbers in parentheses are percentages.

${ }^{*} p$ value $<0.05$

bmp - beats per minute

RT group $(p=0.015)$. Mean effective whole-body dose of a cardiac scan ranged from 0.3 to $3.4 \mathrm{mSv}$.

In total, 3679 coronary artery segments, 849 coronary artery vessels, and 283 patients were evaluated for image quality. Among segments 2538 (69\%) had an image quality score of 4 ("excellent"), 699 segments (19\%) a score of 3 ("good"), 418 segments (11\%) a score of 2 ("moderate"), and 24 segments $(<1 \%)$ were scored as "unevaluable". Mean rating score for all patients and segments was 3.7 ("good" to "excellent"). An example is given in Figure 2. Unevaluable segments were observed in 20 patients. The predominant reasons given by the observers were extensive cardiac motion artefact, noise, and streak artefacts due to extensive calcifications. However, all of these patients belonged to the subgroup that was examined by the "double flash protocol". In these patients, all segments were evaluable after taking both scans into account
Table 3. Distribution of Agaston calcium score

\begin{tabular}{|l|c|}
\hline Total calcium score & $N=256(\%)$ \\
\hline 0 & $24(9.3)$ \\
\hline $1-100$ & $91(35.6)$ \\
\hline $101-200$ & $79(30.9)$ \\
\hline $201-300$ & $30(11.7)$ \\
\hline $301-400$ & $18(7.0)$ \\
\hline$>400$ & $14(5.5)$ \\
\hline
\end{tabular}

(Figure 3). Twenty of these 24 segments were small distal branches with little clinical relevance in the individual patients. The two radiologists demonstrated a good agreement regarding subjective image quality (ICC-coefficient of 0.92).
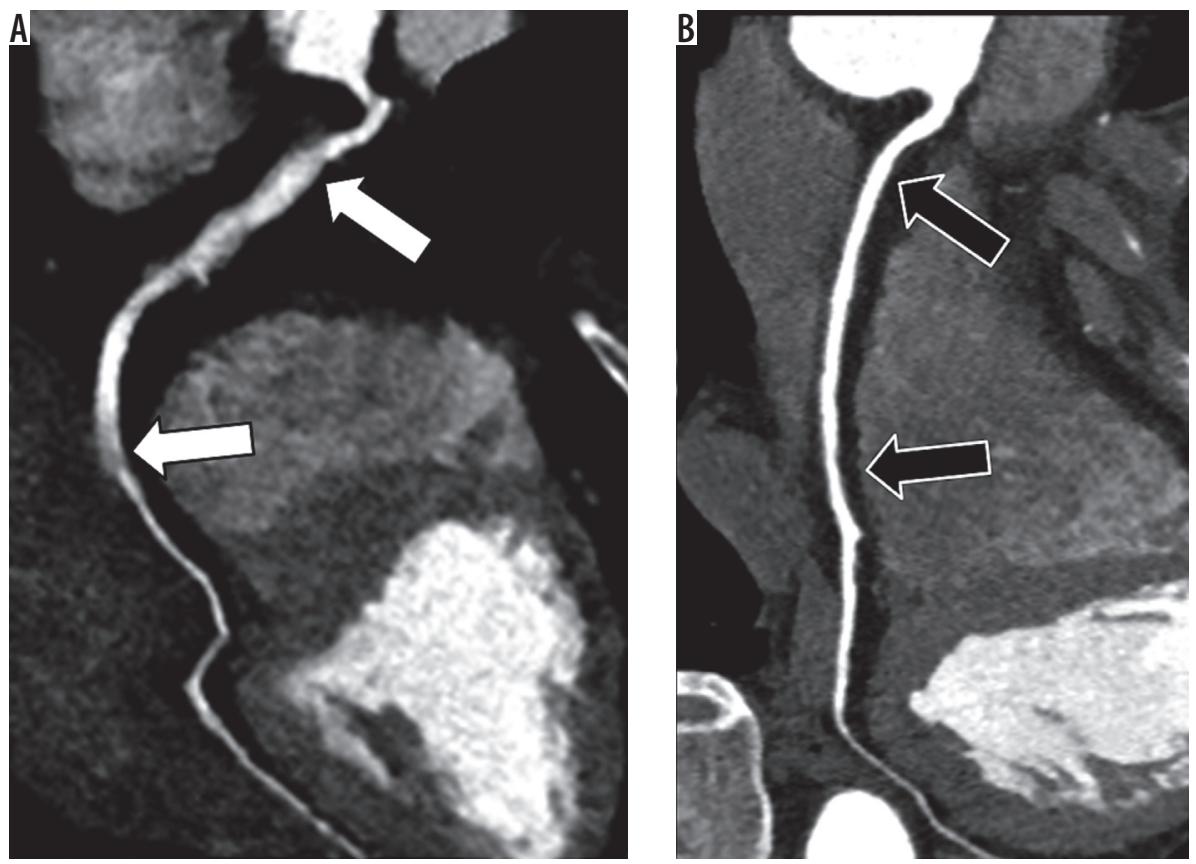

Figure 3. Non-diagnostic image quality ( $A$; white arrow) compared with excellent image quality (B; black arrow) of right coronary artery (RCA) in the same patient after immediate subsequent coronary computed tomography angiography were performed (double flash protocol) 


\section{Discussion}

The use of CCTA with RT results in good image quality and few non-evaluable coronary artery segments; however, the radiation dose to patients is relatively high [27-29].

The second-generation DSCT prospective ECG-triggered high-pitch CCTA offers a unique opportunity to achieve maximal dose reduction while preserving fully diagnostic image quality. DSCT is necessary for this technique because rapid table motion leads to gaps in the trajectory of the first detector. A second detector is needed to fill these gaps. Radiation exposure is very low using this technique since slice overlap (and therefore redundant exposure) is avoided. Using a pitch value of 3.4, data acquisition of the entire $z$-axis of the adult heart within a fraction of one cardiac cycle (approximately $260 \mathrm{~ms}$ ) is possible. Whereas in conventional prospective triggered axial acquisition protocols ("step and shoot") some redundant radiation exposure is still applied at slab interfaces, redundant radiation exposure is nearly eliminated in a high-pitch protocol and occurs only at the beginning and end of the scanned volume [30,31].

The aim of the present study was to describe the results of prospective ECG-triggered high-pitch mode for coronary CTA in clinical routine practice in a tertiary care centre, with respect to image quality and radiation exposure. However, dose reduction must not compromise image quality. Non-diagnostic scans because of 'too little radiation' are a worst-case scenario because they expose the patient to radiation without the hoped for benefit. Several studies have recently described the use of this novel technique $[17,18,32,33]$. Previous studies have shown that prospective ECG-triggered high-pitch spiral acquisition for cardiac CTA is feasible and provides excellent image quality at a consistent dose below $1.0 \mathrm{mSv}$ in carefully selected patients with a low and regular heart rate $(<60 \mathrm{bpm})$ and a body weight of less than $100 \mathrm{~kg}[16,18]$.

We retrospectively analysed 256 patients in which this technique was applied in clinical routine at a tertiary academic centre. In contrast to previous studies, we included all patients scanned on our service regardless of body habitus and with much less rigorous heart rate and rhythm restrictions. Our data show that CCTA using this technique is technically feasible in a less strict clinical routine setting, resulting in an average "good to excellent" image quality at a reasonably low mean radiation exposure. Our studies demonstrated mean radiation dose of $1.6 \mathrm{mSv}$ for all patients and $0.9 \mathrm{mSv}$ for a single-acquisition CCTA, which is in the same range as reported in the previous publications [17,18,32,33].

The percentage of unevaluable segments and subjective image quality analysis was lower in the present study $(0.008 \%)$ compared to the data presented by Lell et al. $(0.6 \%)$ [32]. Nevertheless, the majority of non-diagnostic segments were small, distal, and of little clinical relevance. A wide range of heart rates (range 50-100), excessive cal- cifications of the coronary arteries in bypass graft cases, which lead to "unevaluable" segments due to blooming and streak artefacts. Compared with studies reporting on DSCT of coronary arteries with standard axial "step and shoot" technique, the prospective high-pitch mode technique enables another dose reduction of approximately 40-50\% [13-16]. Earls et al. [16] report a mean effective dose of $2.8 \mathrm{mSv}$ (DLP 170, conversion factor 0.017 ) for a prospective ECG-triggered single-source coronary CTA, whereas an effective dose of $1.2 \mathrm{mSv}$ (DLP 85.7, conversing factor 0.014 ) was found in the study presented herein. Because the radiation dose of a prospective ECG-triggered high-pitch CCTA is very low, a protocol consisting of two scans, one immediately after another ("double flash mode"), might be an opportunity to obtain diagnostic image quality despite a patient's elevated heart rate or occasional PVCs. In comparison to increased "padding" in prospectively triggered single-source CT, which allows assessment of the heart in additional phases within the same cardiac cycle [34], the "double flash protocol" performed in this study concentrates on the same phase $(60 \%)$ at different cardiac cycles.

In the present study, this protocol led to diagnostic image quality of all coronary segments after interpreting both acquisitions. The patients in whom several coronary segments would have been unevaluable after a single scan were diagnostic due to this protocol. Therefore, this technique might obviate a second retrospective ECG-gated cardiac scan with a second injection of contrast, but it still results in low total radiation exposure.

Despite promising initial results, our study has potential limitations. First, our results represent a single-centre experience, the generalisability of the present results is limited. Secondly, a retrospective analysis of clinical data, a systemical bias cannot be excluded. Thirdly, a systematic comparison of CCTA and invasive coronary angiography was not performed, so diagnostic accuracy versus a gold standard is not available. Lastly, radiation doses were calculated and not directly measured in our analysis.

\section{Conclusions}

In the present study, our findings suggest that CCTA performed with prospective ECG-triggered high-pitch is a feasible and promising technique. When applied in clinical routine, evaluation of the coronary arteries is possible at good to excellent image quality with very low radiation exposure. The "double flash protocol" may be a promising technique for patients with higher heart rates $>65 \mathrm{bpm}$ or with irregular heart rates, who might otherwise not receive a diagnosis with a single acquisition in this mode. Further studies are necessary to validate these findings.

\section{Conflict of interest}

The authors report no conflict of interest. 


\section{References}

1. Nikolaou K, Rist C, Wintersperger BJ, et al. Clinical value of MDCT in the diagnosis of coronary artery disease in patients with a low pretest likelihood of significant disease. Am J Roentgenol 2006; 186: 1659-1666.

2. Busch S, Johnson TR, Nikolaou K, et al. Visual and automatic grading of coronary artery stenoses with 64-slice CT angiography in reference to invasive angiography. Eur Radiol 2007; 17: 1445-1451.

3. Johnson TR, Nikolaou K, Busch S, et al. Diagnostic accuracy of dual source computed tomography in the diagnosis of coronary artery disease. Invest Radiol 2007; 42: 684-691.

4. Leschka S, Alkadhi H, Plass A, et al. Accuracy of MSCT coronary angiography with 64-slice technology: first experience. Eur Heart J 2005; 26: 1482-1497.

5. Mollet NR, Cademartiri F, van Mieghem CA, et al. High resolution spiral computed tomography coronary angiography in patients referred for diagnostic conventional coronary angiography. Circulation 2005; $112: 2318-2323$

6. Nikolaou K, Knez A, Rist C, et al. Accuracy of 64-MDCT in the diagnosis of ischemic heart disease. Am J Roentgenol 2006; 187: 111-117.

7. Leber AW, Johnson T, Becker A, et al. Diagnostic accuracy of dualsource multi-slice CT-coronary angiography in patients with an intermediate pretest likelihood for coronary artery disease. Eur Heart J 2007; 28: 2354-2360.

8. Einstein AJ, Henzlova MJ, Rajagopalan S. Estimating risk of cancer associated with radiation exposure from 64-slice computed tomography coronary angiography. JAMA 2007; 298: 317-323.

9. Brenner DJ, Hall EJ. Computed tomography-an increasing source of radiation exposure. N Engl J Med 2007; 357: 2277-2284.

10. Stolzmann P, Scheffel H, Schertler T, et al. Radiation dose estimates in dual-source computed tomography coronary angiography. Eur Radiol 2008; 18: 592-599.

11. Alkadhi H, Stolzmann P, Scheffel H, et al. Radiation dose of cardiac dual-source CT: the effect of tailoring the protocol to patient-specific parameters. Eur J Radiol 2008; 68: 385-391.

12. Scheffel H, Alkadhi H, Leschka S, et al. Low-dose CT coronary angiography in the step-and-shoot mode: diagnostic performance. Heart 2008; 94: 1132-1137.

13. Husmann L, Valenta I, Gaemperli O, et al. Feasibility of low-dose coronary CT angiography: first experience with prospective ECGgating. Eur Heart J 2008; 29: 191-197.

14. Klass O, Jeltsch M, Feuerlein S, et al. Prospectively gated axial CT coronary angiography: preliminary experiences with a novel lowdose technique. Eur Radiol 2008; 19: 829-836.

15. Herzog BA, Husmann L, Burkhard N, et al. Accuracy of low-dose computed tomography coronary angiography using prospective electrocardiogram-triggering: first clinical experience. Eur Heart J 2008; 29: 3037-3042.

16. Earls JP, Berman EL, Urban BA, et al. Prospectively gated transverse coronary CT angiography versus retrospectively gated helical technique: improved image quality and reduced radiation dose. Radiology 2008; 246: 742-753

17. Alkadhi H, Stolzmann P, Baumueller S, et al. Low-dose, 128-slice, dual-source CT coronary angiography: accuracy and radiation dose of the high-patchy and the step-and-shoot mode. Heart 2010; 96 : 933-938.

18. Achenbach S, Marwan M, Ropers D, et al. Coronary computed tomography angiography with a consistent dose below $1 \mathrm{mSv}$ using prospectively electrocardiogram-triggered high-pitch spiral acquisition. Eur Heart J 2010; 31: 340-346.

19. Taylor AJ, Cerqueira M, Hodgson JM, et al. ACCF/SCCT/ACR/ AHA/ASE/ASNC/NASCI/SCAI/SCMR 2010 appropriate use criteria for cardiac computed tomography. A report of the American College of Cardiology Foundation Appropriate Use Criteria Task Force, the Society of Cardiovascular Computed Tomography, the American College of Radiology, the American Heart Association, the American Society of Echocardiography, the American Society of Nuclear Cardiology, the North American Society for Cardiovascular Imaging, the Society for Cardiovascular Angiography and Interventions, and the Society for Cardiovascular Magnetic Resonance. J Am Coll Cardiol 2010; 56: 1864-1894.

20. Raff GL, Abidov A, Achenbach S, et al. Society of Cardiovascular Computed Tomography, SCCT guidelines for the interpretation and reporting of coronary computed tomographic angiography. J Cardiovasc Comput Tomogr 2009; 3: 122-136.

21. Abbara S, Blanke P, Maroules CD, et al. SCCT guidelines for the performance and acquisition of coronary computed tomographic angiography: A report of the Society of Cardiovascular Computed Tomography Guidelines Committee Endorsed by the North American Society for Cardiovascular Imaging (NASCI). J Cardiovasc Comput Tomogr 2016; 10: 435-449.

22. Brodoefel H, Reimann A, Burgstahler C, et al. Noninvasive coronary angiography using 64-slice spiral computed tomography in an unselected patient collective: effect of heart rate, heart rate variability and coronary calcifications on image quality and diagnostic accuracy. Eur J Radiol 2008; 66: 134-141.

23. Ferencik M, Nomura CH, Maurovich-Horvat $\mathrm{P}$, et al. Quantitative parameters of image quality in 64-slice computed tomography angiography of the coronary arteries. Eur J Radiol 2006; 57: 373-379.

24. Mayo JR, Aldrich J, Muller NL. Radiation exposure at chest CT: a statement of the Fleischner Society. Radiology 2003; 228: 15-21.

25. Shrimpton PC, Hillier MC, Lewis MA, Dunn M. National survey of doses from CT in the UK: 2003. Br J Radiol 2006; 79: 968-980.

26. Hausleiter J, Meyer T, Hermann F, et al. Estimated radiation dose associated with cardiac CT angiography. JAMA 2009; 301: 500-507.

27. Hamon M, Biondi-Zoccai GG, Malagutti P, et al. Diagnostic performance of multislice spiral computed tomography of coronary arteries as compared with conventional invasive coronary angiography: a meta-analysis. J Am Coll Cardiol 2006; 48: 1896-1910.

28. Periskinakis K, Seimenis I, Tzedakis A, et al. Triple-rule-out computed tomography angiography with 256-slice computed tomography scanners: patient-specific assessment of radiation burden and associated cancer risk. Invest Radiol 2012; 47: 109-115.

29. Hausleiter J, Meyer T, Hadamitzky M, et al. Radiation dose estimates from cardiac multislice computed tomography in daily practice: impact of different scanning protocols on effective dose estimates. Circulation 2006; 113: 1305-1310. 
30. Leschka S, Scheffel H, Desbiolles L, et al. Image quality and reconstruction intervals of dual-source CT coronary angiography: recommendations for ECG-pulsing windowing. Invest Radiol 2007; 42: 543-549.

31. Wintersperger BJ, Nikolaou K, von Ziegler F, et al. Image quality, motion artifacts, and reconstruction timing of 64-slice coronary computed tomography angiography with 0.33 -second rotation speed. Invest Radiol 2006; 41: 436-442.

32. Lell M, Marwan M, Schepis T, et al. Prospectively ECG-triggered high-pitch spiral acquisition for coronary $\mathrm{CT}$ angiography using dual source CT: technique and initial experience. Eur Radiol 2009; 19: 2576-2583.
33. Goetti R, Leschka S, Baumüller S, et al. Low dose high-pitch spiral acquisition 128-slice dual-source computed tomography for the evaluation of coronary artery bypass graft patency. Invest Radiol 2010; 45: 324-330.

34. LaBounty TM, Leipsic J, Min JK, et al. Effect of Padding Duration on Radiation Dose and Image Interpretation in Prospectively ECG-Triggered Coronary CT Angiography. Am J Roentgenol 2010; 194: 933-937. 